\title{
Medios de comunicación y comportamiento electoral: el referendo sobre la constitución europea en España ${ }^{1}$
}

\author{
ANTONIA M. ${ }^{a}$ RUIZ JIMÉNEZ \\ Departamento de Ciencias Sociales. Área de Sociología \\ Universidad Pablo de Olavide \\ amruiz@upo.es
}

Recibido: 15.10 .2007

Aceptado: 19.12 .2007

\section{INTRODUCCIÓN: EL IMPACTO DE LOS MEDIOS DE COMUNICACIÓN SOBRE LA POLÍTICA Y EL COMPORTAMIENTO ELECTORAL DE LOS CIUDADANOS}

El objetivo principal de este artículo es establecer si el uso que los ciudadanos hicieron de la televisión, la prensa y la radio durante la campaña electoral previa al referendo sobre la constitución europea afectó a su probabilidad de acudir a votar el 20 de febrero de 2005, así como a su probabilidad de votar a favor. Se trata de un trabajo de índole cuantitativa que viene a complementar otros de naturaleza cualitativa, realizados previamente, acerca de la cobertura informativa del referendo sobre la constitución europea en España. Aunque se irán mencionando los aspectos más destacados, es imposible profundizar en sus resultados, por lo que se remite a los lectores interesados a dichos artículos (Sampedro, Ruiz Jiménez y Carriço Reis, 2005; Ruiz Jiménez y Sampedro, 2005; véase también Capdevilla et al., 2005). La pregunta central de esta investigación puede formularse del siguiente modo: ¿Existieron diferencias significativas entre los televidentes de diferentes cadenas, los lectores de diferentes periódicos y los oyentes de diferentes emisoras de radio, en cuanto al

${ }^{1}$ La autora agradece la lectura atenta, comentarios, críticas y sugerencias de Ferrán Martínez i Coma y Víctor Sampedro Blanco, así como el trabajo de los revisores anónimos de la revista Empiria.

EMPIRIA. Revista de Metodología de Ciencias Sociales. N. ${ }^{\circ}$ 14, julio-dicembre, 2007, pp. 13-39.

ISSN: 1139-5737 
porcentaje que acudió a votar y el porcentaje que lo hizo a favor del tratado sobre la constitución europea?².

Existe un debate considerable acerca del impacto de los medios de comunicación sobre el funcionamiento de la democracia. Algunos autores piensan que los éstos juegan un papel positivo, al aportar un flujo de información abundante $\mathrm{y}$ «barata» que los ciudadanos pueden usar para elegir, racionalmente, entre las diferentes ofertas políticas que se les ofrecen. En teoría, los medios de comunicación de masas ofrecen a los ciudadanos información abundante y fácil de conseguir acerca de las ofertas de los partidos, facilitando y promoviendo el debate público sobre diferentes alternativas; por otro lado, ejercerían una función de control sobre los gobiernos que aumentaría la «accountability» de éstos ante la ciudadanía (veánse, entre otros, Swanson y Manzini, 1996; Lupia, 1994; Arnold, 2002). Otros autores, sin embargo consideran que los efectos son negativos; afirman que los medios de comunicación son responsables en buena medida de la alienación, el cinismo, la desconfianza, la desmovilización y la apatía que dominan la política contemporánea (véase Norris, 2000: 4-12 para un resumen de esta línea de argumentación). El presente artículo se inserta en este debate. Se pregunta qué papel jugaron los medios durante la campaña electoral y el referendo sobre la constitución europea: ¿Movilizaron o desmovilizaron los medios a la ciudadanía? ¿Influyeron sobre el voto? ¿Tuvieron todos los medios un efecto similar o diferenciado? ¿En función del tipo de medio o en función de la línea ideológico-editorial?

Antes de responder a estas preguntas, debe plantearse cómo, a través de qué mecanismos, pueden los medios de comunicación influir sobre la ciudadanía y/o sobre su comportamiento electoral. Los primeros estudios sobre esta cuestión se iniciaron de forma paralela al aumento y la circulación de medios de comunicación de masas en las décadas de 1930 y 1940. Estos estudios concluían que los

${ }^{2}$ Podría discutirse si la elección del caso de estudio es acertada o no, ya que tradicionalmente las consultas europeas se han considerado «elecciones de segundo orden» (Reif, 1985; Morata, 2000; Hix, 2001; de Vreese, 2003: 113). En España, además, se ha considerado a la UE más bien como una cuestión transpartidista sobre la que los partidos han tendido a estar de acuerdo (Álvarez Miranda, 1996; Jaúregui, 2001). Esta percepción de la UE como un tema de consenso entre los partidos españoles está siendo revisada sin embargo (Quintanilla Navarro, 2000; Closa, 2001; Torreblanca, 2001; Gómez Reinero et al., 2002; Llamazares, 2005; Ruiz Jiménez y Egea de Haro, 2007 y 2008 -en prensa). Detrás del aparente consenso existen líneas de fractura entre los partidos tanto en la dimensión ideológica como en el eje centro-periferia.

Respecto a estos argumentos cabe señalar, en primer lugar, que la baja participación en una consulta electoral no la descalifica como objeto de estudio, aunque haga más difícil la aplicación de técnicas de análisis multivariantes. Por otro lado, no se está analizando el resultado de unas elecciones al Parlamento Europeo, sino de un referéndum lo que quizás no hace completamente aplicable las conclusiones alcanzadas por otros autores respecto a las primeras. Por último, de los estudios cualitativos realizados sobre el tema del referendo se desprende que, efectivamente, hubo líneas de fractura entre los tanto en la dimensión ideológica como en el eje centro-periferia (Sampedro, Ruiz Jiménez y Carriço Reis, 2005; Ruiz Jiménez y Sampedro, 2005; véase también Capdevilla et al., 2005; y Ruiz Jiménez y Noya, 2004). En este sentido, lo que el artículo se propone analizar es si dichos mensajes llegaron a la ciudadanía y qué efectos tuvieron sobre su comportamiento electoral.

EMPIRIA. Revista de Metodología de Ciencias Sociales. N. ${ }^{\circ}$ 14, julio-dicembre, 2007, pp. 13-39. ISSN: 1139-5737 
efectos de los medios de comunicación sobre el voto eran poco importantes debido a que los ciudadanos prestaban una atención selectiva a la propaganda electoral (Lazarsfeld, Berelson y Gaudet, 1944; Berelson, Lazarsfeld y MacPhee, 1954; Patterson y MacClure, 1976) ${ }^{3}$.

Investigaciones más recientes, llevadas a cabo desde finales de los años 1990, vienen a demostrar que los medios de comunicación tienen efectos mayores de lo que se pensaba sobre la opinión pública y el comportamiento electoral, aunque éstos no se produzcan de forma directa ${ }^{4}$.

Se habla, entonces, «efectos de segundo orden» (o indirectos), cuya influencia sobre persuasión política no debe ser menospreciada sin embargo (Swanson y Mancini, 1996; Iyengar y Reeves, 1997; Entman 1993). Dicha influencia se produciría a través de procesos como el la fijación de la agenda (agenda-setting), la intensificación de mensajes (priming) y su enmarcado (framing).

Más recientemente, algunos autores han vuelto a plantear de nuevo a la idea de que los medios de comunicación modernos pueden un efecto directo sobre las opiniones políticas y el comportamiento electoral de los ciudadanos, incluso mayor de lo que se cree (véanse, entre otros: Zaller, 1996; Dalton, Beck y Huckfield, 1998; Newton y Brynin, 2001; Brynin y Newton, 2003). En el caso de España, los estudios son más escasos y los resultados menos claros. Mientras que Fernández Mellizo-Soto (2000) no encuentra que los medios de comunicación tuvieran efecto sobre los resultados electorales de 1993, Schmitt-Beck (2003) encuentra evidencia de lo contrario, y para la misma consulta electoral de 1993. Véanse también los estudios de Muñoz Alonso y Rospir (1999) y Crespo Martínez (2002-2004) para posteriores campañas electorales.

De forma más específica, distintos investigadores han indagado también en el impacto de los medios de comunicación sobre la opinión pública acerca de la UE. A nivel internacional, Banducci y Semetko (2003) han probado el impacto del volumen y el tono de la cobertura de la EU en los medios de comunicación sobre la probabilidad de acudir a votar en las elecciones europeas. En el caso específico del Reino Unido, Carey y Burton (2004) han demostrado que la prensa influye en las actitudes de los británicos sobre la Unión Europea, aún después de tener en cuenta el efecto de otras variables socio-demográficas, la identificación partidista de los votantes, y sus consideraciones instrumentales sobre la UE. No quiere esto decir que el impacto sea necesariamente positivo (véase Norris et al., 1999).

${ }^{3}$ Es decir, los votantes seleccionaban los mensajes a los que prestar atención en función de la decisión que habían tomado previamente respecto a cual sería el sentido de su voto (en función de su preferencia partidista), por lo que los medios tendrían sólo un efecto mínimo de refuerzo sobre las opiniones de los ciudadanos. En resumen, serían otras variables (ideología, educación, clase social...) las que afectarían simultáneamente a la preferencia partidista y la elección de un medio de comunicación.

${ }^{4}$ También se ha ampliado el concepto de comportamiento electoral, incluyendo otras manifestaciones además del ejercicio directo del voto.

EMPIRIA. Revista de Metodología de Ciencias Sociales. N. ${ }^{\circ}$ 14, julio-dicembre, 2007, pp. 13-39.

ISSN: $1139-5737$ 
El presente trabajo se inserta en esta línea de investigación más reciente. Trata de comprobar el efecto directo que el consumo de diferentes medios de comunicación pudo tener sobre el comportamiento electoral de los ciudadanos españoles en el referendo sobre la constitución europea. Para salvar parte de las dificultades metodológicas que este tipo de investigación plantea, se ha recurrido a métodos de análisis multivariantes, incluyendo en ellos todas las variables que pudieran haber influido sobre dicho comportamiento electoral además de la exposición de los ciudadanos a determinados medios de comunicación. Dadas las dificultades de este tipo de estudio, el siguiente epígrafe se dedica a aclarar cuestiones metodológicas.

\section{2. ¿QUÉ INFLUYE SOBRE QUÉ? PROBLEMAS DE AUTO- SELECCIÓN Y SOLUCIONES METODOLÓGICAS DE LA INVESTIGACIÓN}

El problema principal con el que se encuentra cualquier investigación que pretenda indagar sobre las relaciones causales entre el consumo de medios de comunicación por parte de los ciudadanos y su comportamiento electoral, es que ambos aspectos están estrechamente conectados con otras variables de orden social, económico y político. Así, el nivel educativo, la clase social, la ocupación, el nivel de ingresos o la edad, están estrechamente ligados con el consumo de determinado tipo de medio de comunicación, por un lado, y con las actitudes políticas y el comportamiento electoral, por el otro, haciendo muy difícil separar los efectos de estas variables socio-demográficas de los efectos de los medios de comunicación sobre las actitudes políticas y el comportamiento electoral de los ciudadanos $^{5}$. Por poner un ejemplo, en el caso concreto de este artículo, es posible que la ideología de izquierda de algunos entrevistados explique simultáneamente su preferencia por la lectura de El País, su voto al PSOE en las elecciones generales de 2004 y su decisión de acudir a votar el día del referendo sobre la constitución europea.

Una posible solución a este problema metodológico de auto-selección de los entrevistados consiste en llevar a cabo análisis multivariantes que controlen el efecto de esas otras variables. Si aún controlando el efecto de las variables sociodemográficas y las preferencias partidistas, encontramos que los medios influyen

${ }^{5}$ Un problema adicional, y quizás de más difícil solución, lo constituye el hecho de que la relación de causa-efecto entre los medios de comunicación y sus audiencias no está clara. Las audiencias seleccionan los medios a los que prestarán atención, tanto como los medios intenta captar un tipo de audiencia determinada a la que debe ofrecer el producto que ésta desea recibir. Visto de este modo, y en la medida en que los medios se ven influidos por las fuerzas del mercado, éstos reflejarían, se harían eco, y, de hecho, seguirían a la opinión pública, más de lo que la moldearían, influenciarían o llegarían a cambiarla (véanse: Newton y Brynin, 2001: 266) ; Seymour-Ure, 1997). A efectos prácticos, en este capítulo se asume, y comprueba, la relación causal de los medios sobre la opinión pública.

EMPIRIA. Revista de Metodología de Ciencias Sociales. N. ${ }^{\circ}$ 14, julio-dicembre, 2007, pp. 13-39. ISSN: 1139-5737 
sobre el comportamiento electoral de los ciudadanos, las conclusiones serán robustas. Por otro lado, es posible comparar a aquellos ciudadanos que consumen medios de comunicación con una línea ideológico-editorial congruente con la del partido al que se sienten más cercanos (aquellos en los que puede estar funcionando la auto-selección) con quienes consumen medios de comunicación cuya línea ideológico-editorial no es congruente con la del partido al que se sienten más cercanos (en los que la auto-selección no se produce, ya que eligen medios que no se adecuan a sus preferencias ideológicas). De este modo, las variaciones que se observen en el comportamiento electoral de estos dos grupos (controlando en ambos casos por el resto de variables socio-demográficas mencionadas) deberían ser atribuidas al cambio en la prensa consumida. Se sigue, así, una metodología similar a la utilizada en estudios recientes por Newton y Brynin (2001), Brynin y Newton (2003), Schmitt-Beck (2003), y Carey y Burton (2004).

Para aplicar este tipo de análisis al caso español es necesario contar con una base de datos que contenga todas las variables necesarias para realizarlo, en la cual exista, además, un número suficiente de casos de personas que consumen medios de comunicación teóricamente no congruentes con sus preferencias partidistas. Afortunadamente contamos con la octava oleada del Barómetro del Real Instituto Elcano ${ }^{6}$, cuyo trabajo de campo se realizó entre el 22 de febrero y el 3 de marzo de 2005, y en cuyo cuestionario se incluían cuestiones relativas al voto de los entrevistados en el referendo sobre la constitución europea (en febrero de 2005), así como preguntas relativas a su utilización y consumo de los medios de comunicación y al partido al que votaron en las elecciones generales de 2004. El barómetro contenía, por lo demás, el conjunto de variables socio-demográficas habitual, la cuales se han utilizado como variables de control en los modelos.

Utilizando estos datos, puede comprobarse en primer lugar que, efectivamente, existe una relación estadísticamente significativa entre el partido al que los ciudadanos votaron en las elecciones generales de 2004 y su preferencia por determinados medios de comunicación (cadenas de televisión, periódicos, o emisoras de radio). Así, quienes votan a partidos de izquierda seleccionan habitualmente medios de comunicación también de izquierdas para informarse, en mayor proporción, en cualquier caso, de lo que cabría esperar (si ambas selecciones fueran independientes entre sí) (véanse Tablas 1, 2 y 3).

${ }^{6}$ Universo: Población de ambos sexos de 18 años y más. Ámbito: Nacional, incluyendo Ceuta y Melilla. Tamaño muestral: $\mathrm{N}=1.200$ individuos. Técnica: CATI, entrevista telefónica asistida por ordenador (llamada al hogar del entrevistado). Diseño de la muestra: Polietápica estratificada. Afijación proporcional por el doble criterio Comunidad Autónoma-hábitat proporcional a la población de cada CA, aplicándose a la unidad última (individuo) cuotas de sexo y edad. Error muestral: $\pm 2,9 \%$ (1.200n) para datos globales, $p=q=0,5$ y un intervalo de confianza del 95,5\%. Fecha de campo: del 22 de febrero al 3 de marzo de 2005. Trabajo de campo: TNS-Demoscopia.

A pesar de que el estudio incluye muchas de las variables necesarias para poder realizar el análisis, cabe lamentar la ausencia de otras que hubieran mejorado la calidad del análisis y las conclusiones, tales como la frecuencia con que se leen las secciones de política de la prensa escrita, o se atiende a programas de radio o televisión con contenidos políticos. Con todo, es el estudio más completo que se ha podido encontrar para llevar a cabo el análisis.

EMPIRIA. Revista de Metodología de Ciencias Sociales. N. ${ }^{\circ}$ 14, julio-dicembre, 2007, pp. 13-39.

ISSN: 1139-5737 
Tabla 1. Porcentaje de votantes de cada partido que mira diferentes cadenas de televisión, en función de su línea ideológico-editorial*

\begin{tabular}{|c|c|c|c|c|c|}
\hline & $\begin{array}{l}\text { Izquierda } \\
\text { (a) }\end{array}$ & $\begin{array}{l}\text { Derecha } \\
\text { (b) }\end{array}$ & $\begin{array}{l}\text { Autonómica/ } \\
\text { local }\end{array}$ & Ninguna & $\begin{array}{l}\text { Votantes } \\
\text { incluidos }\end{array}$ \\
\hline PSOE & $69(4,9)$ & $18(-2,5)$ & $10(-3,9)$ & 4 (n.s.) & 371 \\
\hline PP & $53(-2,0)$ & $34(5,3)$ & $10(-3,0)$ & 3 (n.s.) & 267 \\
\hline IU & 65 (n.s.) & $8(-2,5)$ & 22 (n.s.) & 6 (n.s.) & 51 \\
\hline $\begin{array}{l}\text { Ámbito } \\
\text { Autonómico SI (c) }\end{array}$ & $26(-4,4)$ & 23 (n.s.) & $51(6,4)$ & - & 43 \\
\hline $\begin{array}{l}\text { Ámbito } \\
\text { autonómico NO (d) }\end{array}$ & $38(-2,7)$ & 12 (n.s.) & $50(6,1)$ & - & 42 \\
\hline
\end{tabular}

* Los números en las casillas son porcentajes de fila provenientes de análisis de tablas de contingencia. Se han incluido los residuos estandarizados corregidos entre paréntesis. Sólo se han incluido las diferencias estadísticamente significativas (mayores de 2) entre la frecuencia esperada y la frecuencia observada.

(a) TVE, Tele 5, Canal Plus.

(b) Antena 3.

(c) Partidos de ámbito autonómico que hicieron campaña a favor del Sí: PNV, CiU, CC.

(d) Partidos de ámbito autonómico que hicieron campaña a favor del No: BNG, CHA, EA, ERC

Medidas de asociación: Phi, 340***, V de Cramer, 196***.

Chi cuadrado de Pearson: 134,190***.

FUENTE: BRIE $8 .^{\mathrm{a}}$ oleada.

Tabla 2. Porcentaje de votantes de cada partido que lee diferentes periódicos, en función de su línea ideológico-editorial*

\begin{tabular}{lcccccc}
\hline & $\begin{array}{c}\text { Izquierda } \\
(\mathbf{a})\end{array}$ & $\begin{array}{c}\text { Derecha } \\
(\mathbf{b})\end{array}$ & $\begin{array}{c}\text { Otros } \\
\text { pago y/o } \\
\text { gratuitos }\end{array}$ & $\begin{array}{c}\text { Autonó- } \\
\text { mica/ } \\
\text { local }\end{array}$ & Ninguna & $\begin{array}{c}\text { Votantes } \\
\text { incluidos }\end{array}$ \\
\hline PSOE & $27(5,6)$ & $7(-6.1)$ & 8 (n.s.) & 38 (n.s.) & 20 (n.s.) & 376 \\
PP & $8(-4,8)$ & $26(4,8)$ & 7 (n.s.) & 40 (n.s.) & 19 (n.s.) & 270 \\
IU & $41(4,4)$ & 12 (n.s.) & 10 (n.s.) & 28 (n.s.) & 10 (n.s.) & 51 \\
$\begin{array}{l}\text { Ámbito } \\
\begin{array}{l}\text { Autonómico } \\
\text { SI (c) }\end{array}\end{array}$ & $2(-2,8)$ & $13(2,4)$ & 14 (n.s.) & 48 (n.s.) & $7(-2,0)$ & 44 \\
$\begin{array}{l}\text { Ámbito } \\
\text { autonómico } \\
\text { NO (d) }\end{array}$ & - & 19 (n.s.) & 5 (n.s.) & $62(3,1)$ & 14 (n.s.) & 42 \\
\hline
\end{tabular}

* Los números en las casillas son porcentajes de fila provenientes de análisis de tablas de contingencia. Se han incluido los residuos estandarizados corregidos entre paréntesis. Sólo se han incluido las diferencias estadísticamente significativas (mayores de 2) entre la frecuencia esperada y la frecuencia observada.

(a) El País.

(b) El Mundo, ABC, La Vanguardia.

(c) Partidos de ámbito autonómico que hicieron campaña a favor del Sí: PNV, CiU, CC.

(d) Partidos de ámbito autonómico que hicieron campaña a favor del No: BNG, CHA, EA, ERC.

Medidas de asociación: Phi, 336***, V de Cramer, 168***.

Chi cuadrado de Pearson: 133,271 ** .

FUENTE: BRIE 8. ${ }^{\mathrm{a}}$ oleada. 
Tabla 3. Porcentaje de votantes de cada partido que escucha diferentes emisoras de radio, en función de su línea ideológico-editorial*

\begin{tabular}{|c|c|c|c|c|c|}
\hline & $\begin{array}{l}\text { Izquierda } \\
\text { (a) }\end{array}$ & $\begin{array}{l}\text { Derecha } \\
\text { (b) }\end{array}$ & Otras & Ninguna & $\begin{array}{l}\text { Votantes } \\
\text { incluidos }\end{array}$ \\
\hline$\overline{\text { PSOE }}$ & $47(6,4)$ & $10(-5,3)$ & $13(-2,0)$ & 30 & 374 \\
\hline PP & $22(-4,7)$ & $40(2,0)$ & $12(-2,4)$ & $27(-2,0)$ & 267 \\
\hline IU & $49(2,4)$ & $8(-2,0)$ & 12 & 31 & 51 \\
\hline $\begin{array}{l}\text { Ámbito } \\
\text { Autonómico SI (c) }\end{array}$ & 25 & $2(-2,8)$ & $34(3,2)$ & 39 & 44 \\
\hline $\begin{array}{l}\text { Ámbito } \\
\text { autonómico NO (d) }\end{array}$ & 34 & 12 & $42(4,4)$ & $12(-2,7)$ & 41 \\
\hline
\end{tabular}

* Los números en las casillas son porcentajes de fila provenientes de análisis de tablas de contingencia. Se han incluido los residuos estandarizados corregidos entre paréntesis. Sólo se han incluido las diferencias estadísticamente significativas (mayores de 2) entre la frecuencia esperada y la frecuencia observada.

(a) La Ser, Radio Nacional.

(b) La COPE, Onda O.

(c) Partidos de ámbito autonómico que hicieron campaña a favor del Sí: PNV, CiU, CC.

(d) Partidos de ámbito autonómico que hicieron campaña a favor del No: BNG, CHA, EA, ERC.

Medidas de asociación: Phi, 390***, V de Cramer, 225***.

Chi cuadrado de Pearson: 178,954***.

FUENTE: BRIE $8 .^{\text {a }}$ oleada.

En otras palabras, observamos cierta auto-selección en la medida en que los ciudadanos escogen medios de comunicación que son congruentes con su ideología, por lo que podría anticiparse que el efecto de los medios fuera mínimo sobre el comportamiento electoral en el referendo sobre la constitución europea (una vez que controláramos el efecto de la preferencia electoral). No obstante, cabe señalar la existencia de algunos porcentajes considerables de ciudadanos que, en las Tablas 1, 2 y 3, seleccionan medios de comunicación cuya línea ideológico-editorial no es congruente con la preferencia partidista mostrada en las elecciones generales de 2004 (es decir, que votaron a un partidos de izquierda pero seleccionaron un medio de derechas, o viceversa). Así, un $18 \%$ de quienes votaron al PSOE miran Antena 3 habitualmente, en tanto que un $22 \%$ de quienes votaron al PP escuchan Radio Nacional o la SER, por ejemplo. Se puede hipotetizar que estos ciudadanos habrían recibido mensajes poco congruentes de sus partidos y de los medios de comunicación a través de los cuales siguieron la campaña del referendo sobre la constitución europea ${ }^{7}$. Existen varias razones por las que los ciudadanos pueden elegir medios de comunicación que no se ajusten a su preferencia partidista. Es posible, en primer lugar, que los entrevistados seleccionen los medios de comunicación en función de otros criterios que no sean políticos, o al menos no exclusivamente políticos, tales como el entreteni-

${ }^{7}$ Más adelante se hará referencia explícita acerca de la dirección en la que se produjeron, o no, mensajes de refuerzo desde los partidos y los medios de comunicación.

EMPIRIA. Revista de Metodología de Ciencias Sociales. N. ${ }^{\circ}$ 14, julio-dicembre, 2007, pp. 13-39.

ISSN: 1139-5737 
miento, la cobertura deportiva, los suplementos dominicales, porque es el medio que consumen también su pareja o sus amigos, por su fama de independiente y/o fiable, o, incluso a sabiendas de que no es el que mejor refleja sus propias preferencias ideológicas, porque se desea conocer el mensaje del adversario político. En segundo lugar, es probable que un porcentaje importante de ciudadanos no tenga una clara conciencia de cuál es la ideología que se supone que reflejan determinados medios de comunicación ${ }^{8}$. Por último, es posible que algunos no tengan muy clara su propia preferencia ideológica (a pesar de que votaran a un determinado partido en las elecciones generales de 2004) ${ }^{9}$. Sea cual sea el motivo, el hecho es que existe un porcentaje de entrevistados que recibe mensajes poco congruentes de su partido y de los medios de comunicación que utilizan (debido a sus líneas ideológicas divergentes), por lo que, en principio, será posible ver si los medios tienen un impacto sobre el comportamiento electoral de los ciudadanos y distinguir ese impacto de la influencia de los partidos y del resto de variables.

\section{ANÁLISIS: ¿QUÉ OCURRIÓ EN EL REFERENDO SOBRE LA CONSTITUCIÓN EUROPEA EN ESPAÑA?}

\section{Hipótesis}

En función de lo expuesto hasta ahora cabe formular algunas hipótesis concretas respecto a la influencia de los medios de comunicación sobre el comportamiento electoral de los ciudadanos españoles en el referendo sobre la constitución europea, que se tratarán de comprobar en los apartados siguientes.

En primer lugar, cabe esperar que quienes utilizan habitualmente algún medio de comunicación acudieran a votar en mayor medida que quienes normalmente no lo hacen. Quienes vieron la televisión, leyeron la prensa u oyeron la radio durante el periodo de campaña electoral tuvieron una probabilidad más alta de recibir información sobre ella que quienes no lo hicieron. Cuanto más visible fuera la campaña para los ciudadanos, más probable es que percibieran que el referendo sobre la constitución europea y su voto en él (ya fuera este positivo o negativo) eran importantes. Desde esta perspectiva los medios pueden incrementar los porcentajes de voto en las consultas electorales au-

\footnotetext{
${ }^{8}$ Algo más frecuente en el caso de la TV que de los periódicos, más marcados por alineamientos editorial-partidarios, y que encuentra cierto reflejo en las tablas 1 y 2.

${ }^{9}$ De hecho, en el Barómetro del Real Instituto Elcano que se usa en este capítulo era mayor el número de personas que no contestan a la pregunta sobre su ideología (555 entrevistados, un $46 \%$ de la muestra) que a la pregunta sobre recuerdo de voto (223, un $19 \%$ de la muestra). Por el contrario, prácticamente todos los entrevistados contestaron a la pregunta sobre la cadena de televisión en que veían las noticias, la prensa que leían para informarse, o la emisora de radio que escuchaban; no respondieron a esas pregunta 52,18 y 26 personas $(4 \%, 1,8 \%$ y $2,1 \%$ de la muestra) respectivamente.
} 
mentando tanto la percepción de los beneficios de ir a votar (la percepción de que la elección y el propio voto son importantes), como la percepción acerca de los costes que implicaría no ir a votar (Banducci y Semetko, 2003: 2; Banducci et al., 2004: 6).

En segundo lugar, y puesto que la probabilidad de recibir información sobre el referendo y la constitución europea sería una función tanto de la visibilidad del mensaje en los medios como de la frecuencia con que los ciudadanos se conectaron a la fuente de información (Zaller, 1992), cabe esperar que quienes utilizaron medios cuya cobertura sobre el referendo fue más amplia acudieran a votar con una probabilidad más alta que quienes utilizaron medios cuya cobertura fue más limitada. Podemos concretar esta hipótesis para la prensa: quienes leyeron El País, el periódico con más informaciones sobre la constitución (véanse: Sampedro, Ruiz Jiménez y Carriço Reis, 2005; Ruiz Jiménez y Sampedro 2005), acudieron a votar en mayor proporción que quienes no leen habitualmente ningún periódico, o quienes leyeron $E l$ Mundo o $A B C^{10}$.

En tercer lugar, si se incorpora el efecto de refuerzo de los mensajes en las hipótesis, podría esperarse que quienes votaron al PSOE en 2004 y leyeron $E l$ País para informarse sobre la campaña del referendo sobre la constitución europea acudieran a votar en mayor medida que quienes votaron al PSOE en 2004, pero leyeron otros periódicos, como El Mundo o $A B C$. Lo mismo cabría señalar con respecto a diferentes canales de televisión o emisoras de radio, aunque no se cuenta con información específica acerca de su cobertura de la campaña electoral. No obstante, dadas las tendencias ideológico-editoriales de cada medio, los mensajes de refuerzo se producirían teóricamente entre los votantes del PSOE que son también televidentes de TVE, Tele 5 o Canal + , y quienes son oyentes de Radio Nacional y la SER; entre los votantes del PP habrían recibido mensajes congruentes (aumentando el refuerzo) los televidentes

${ }^{10}$ Entre el 11 de octubre de 2004 y el 28 de febrero de 2005, aparecieron en El País 271 unidades de análisis relacionadas con el referéndum sobre la constitución europea, frente a las 171 de $A B C$ y las 137 de El Mundo. La siguiente cita resume, además, las conclusiones del análisis cualitativo respecto a la cobertura general en los tres periódicos (Sampedro, Ruiz Jiménez y Carriço Reis 2005: 7):

(...) Mientras todos los diarios señalaban el grado de desconocimiento, El País enfatizó que ello no impediría un alto grado de participación y respaldo popular. Por su parte, $A B C$ y $E l$ Mundo resaltaban que el resultado positivo de la consulta se produciría a pesar de la ignorancia y la indiferencia ante el Tratado. Es decir, mientras la cobertura del primer diario se teñía de optimismo, los dos últimos señalaban las contradicciones de la consulta e, incluso, insinuaban su falta de legitimidad, subrayando el escaso grado de conocimiento y la apatía existentes. Destaca que sólo El País recogiese la división de opiniones (brindando razones para continuar la campaña) y que sólo El Mundo señalase la existencia de posiciones contrarias a la Constitución europea. Las referencias al «no», sumadas a la mayor presencia de informaciones sobre el desinterés ciudadano, doblaban en número a las noticias sobre el respaldo popular. El balance final de este diario, como $A B C$, era por tanto de subrayar las actitudes ciudadanas más negativas, frente a la confianza con la que El País interpretaba el clima de opinión previo al referendo.

EMPIRIA. Revista de Metodología de Ciencias Sociales. N. ${ }^{\circ}$ 14, julio-dicembre, 2007, pp. 13-39. ISSN: $1139-5737$ 
de Antena 3 y los oyentes de Onda 0 y la COPE. No obstante, dada la cobertura de la campaña del referendo, al menos por lo que hace a la prensa, cabría esperar que los votantes del PP que leyeron El País durante la campaña electoral, acudieran a votar en mayor medida que quienes leyeron El Mundo o $A B C$, ya que como se ha señalado en trabajos anteriores, el referendo recibió mayor cobertura en El País, lanzando además un mensaje más claro respecto a la necesidad de acudir a votar que los otros dos periódicos, mensaje que era, al menos en el nivel más superficial, congruente con la postura del PP en el referendo.

Por lo que hace al sentido del voto, cabría esperar, en cuarto lugar, que quienes votaron al PSOE en 2004 y leyeron El País, recibiendo de este modo mensajes congruentes respecto al sentido esperado de su voto, hubieran emitido un voto favorable en mayor proporción que quienes votaron al PSOE en 2004 pero leyeron otros periódicos, como $A B C$ o $E l M u n d o$, en los que el mensaje favorable no aparecía tan frecuentemente ni de forma tan obvia, aunque predominara, ya que el apoyo al borrador de constitución europea era también la postura oficial del $\mathrm{PP}^{11}$. Como se indicaba también más arriba, aunque no existe de un estudio detallado de la cobertura del referendo en la televisión y la radio, podemos formular hipótesis acerca de qué votantes recibieron mensajes congruentes. Así, se habría producido un efecto de refuerzo de mensajes entre los votantes del PSOE que son también televidentes de TVE, Tele 5 o Canal + , y quienes son oyentes de Radio Nacional y la SER; entre los votantes del PP habrían recibido mensajes congruentes (aumentando el refuerzo) los televidentes de Antena 3 y los oyentes de Onda 0 y la COPE. No obstante, dada la cobertura de la campaña del referendo, al menos por lo que hace a la prensa, cabe esperar que los votantes del PP que leyeron El País durante la campaña electoral, votaran a favor en mayor medida que quienes leyeron $E l$ Mundo o $A B C$, ya que como se señalado en trabajos previos, esta postura recibía mayor cobertura en El País, lanzando además un mensaje más claro tanto con respecto a la necesidad de votar a favor, como respecto a los costes que tendría el voto negativo en el referendo. Aunque oficialmente la postura del PP era el voto a favor, quienes leyeron El País se vieron expuestos a este mensaje con mayor frecuencia que quienes leyeron $E l$ $M u n d o$ y $A B C$, donde el mensaje era menos obvio.

\footnotetext{
${ }^{11}$ No existe una variable en la base de datos que mida la «congruencia». Por el contrario se ha estimado que existe congruencia en los mensajes que recibe un votante, si la ideología del medio de comunicación seleccionado coincide con la ideología del partido al que votó en las elecciones generales de 2004. Cabría señalar que el partido al que se votó en las elecciones de 2004 puede no ser un buen predictor del voto en el referéndum sobre la constitución europea, ya que los condicionantes que determinan el voto son diferentes según el nivel (local, autonómico, nacional o supranacional) de la consulta - véase la nota 2 del artículo. No obstante, el partido al que se votó en las elecciones de 2004 se utilizan como «proxy», ya que se carece de información en la base de datos respecto al partido al que se votó en las anteriores elecciones europeas.
}

EMPIRIA. Revista de Metodología de Ciencias Sociales. N. ${ }^{\circ}$ 14, julio-dicembre, 2007, pp. 13-39. ISSN: 1139-5737 


\section{Especificación de los modelos}

Como se deduce de las hipótesis anteriores, el análisis se centra en dos variables dependientes: la probabilidad de haber acudido a votar el día del referendo sobre la constitución europea, y la probabilidad de haber votado a favor. Ambas se miden como variable dicotómicas, en las que el valor uno indica que se acudió a votar o se votó a favor, en cada una de las variables respectivamente, y el valor cero que no se hizo (es decir, que no se acudió a votar o que se votó en contra respectivamente); el resto de posibilidades se consideran valores perdidos en los análisis.

En cuanto a las variables independientes, o explicativas, se han tomado en consideración tanto aquellas que se ha demostrado que tienen influencia sobre el comportamiento electoral en general, como las que se piensa que afectan específicamente al apoyo a la integración europea, ya que la decisión de acudir a votar en el referendo y, especialmente, el voto favorable al borrador de tratado pueden interpretarse también como una forma de apoyo a la integración europea, al menos al proyecto reflejado en el borrador de constitución.

Entre las variables independientes de los modelos se han incluido, en primer lugar, el recuerdo de voto en las elecciones de 2004. Esta variable se utiliza como indicador de la preferencia partidista de los ciudadanos, asumiendo que votaron al partido al que se sentían más cercanos y/o que representaba mejor sus intereses o sus preferencias ideológicas, ya que carecemos de una variable que mida específicamente la preferencia partidista de los entrevistados. Cabe esperar que, si los ciudadanos utilizan sus preferencias partidistas como atajos (o proxies) para tomar decisiones políticas (Anderson, 1998), votaran en el referendo sobre la constitución europea de acuerdo a las consignas del partido al que habían votado en las elecciones generales de 2004.

En segundo lugar se tiene en cuenta el medio de comunicación a través del cual los entrevistados se informan habitualmente, y a través del cual se informaron, por tanto, durante la campaña electoral. Ya se ha referido, en una sección anterior al impacto que los medios de comunicación pueden tener sobre la democracia, el comportamiento electoral de los ciudadanos, y, de forma aún más concreta, sobre las actitudes y el apoyo a la integración europea. También se han hecho explícitas las hipótesis respecto a la influencia que se espera que tuvieran sobre el comportamiento electoral en el referendo sobre la constitución europea.

A estas variables se han añadido el nivel educativo de los entrevistados. Desde la óptica instrumental que ha predominado en los estudios sobre apoyo a la integración europea, el nivel educativo se ha tomado como indicador del capital humano de los ciudadanos. Autores como Gabel (1998a y 1998b) han señalado que aquellos ciudadanos con mayor nivel educativo están mejor capacitados para adaptarse a los cambios económicos provocados por la liberalización del mercado de trabajo dentro de la UE. La educación es, también, una de las variables socio-económicas con mayor poder explicativo del comportamiento elec- 
toral de los ciudadanos españoles (Lancaster y Lewis-Beck, 1986; González, 1993; Fernández Mellizo-Soto, 2000; Crespo Martínez, 2002-2004) ${ }^{12}$.

En la misma línea de apoyo instrumental a la UE, Gabel (1998a y 1998b) ha señalado que los ciudadanos de determinados sectores económicos resultan afectados de forma diferente por el proyecto de integración. En los modelos se ha incluido, por tanto, la situación profesional de los ciudadanos, tomando a las amas de casa como grupo de referencia.

Se han añadido, además, la edad, el sexo y el tamaño del núcleo de residencia de los entrevistados. Por lo que respecta a la edad, algunos autores han señalado que los jóvenes tienen una percepción más cosmopolita del mundo y de su propia identidad, por lo que se sentirían más inclinados a apoyar el proyecto de integración europea (Inglehard y Rabier, 1978). En cuanto al sexo, algunos estudios apuntan a que las mujeres se encuentran entre las «perdedoras» del proyecto de integración, por lo que tenderían a apoyar la UE en menor medida que los hombres (Gelleny y Anderson, 2000: 185). Finalmente, es posible que el tamaño del núcleo de residencia pueda afectar a las actitudes y comportamiento de los entrevistados si quienes viven en aglomeraciones urbanas reciben más información y mantienen más contactos internacionales (lo que influiría en una visión más cosmopolita), que quienes habitan en áreas rurales ${ }^{13}$.

Cabría considerar, en última instancia, la identidad de los entrevistados. El predominio de las identidades nacionales se ha relacionado en algunos países con una probabilidad mayor de rechazo a la integración europea, en tanto que las identidades sub-nacionales (o nacionalistas) podrían relacionarse con un mayor apoyo a la UE (véase Diez-Medrano y Gutierrez, 2001). Sin embargo, la forma en que esta variable está medida en el barómetro que se utiliza para los análisis no era adecuada ni resultaba significativa, por otro lado, cuando se introducía en los modelos. En su lugar, se ha optado por incluir la Comunidad autónoma, que junto con la preferencia partidista de los entrevistados, puede capturar adecuadamente la existencia o no de identidades nacionalistas.

${ }^{12}$ Por otro lado, la educación se relaciona muy estrechamente con el nivel de ingresos de los ciudadanos y con su clase social, variables con un impacto considerable también sobre el comportamiento electoral (González Álvarez, 1998). El nivel de ingresos, sin embargo, no se incluía en el cuestionario del barómetro que utilizamos en este estudio. La clase social se incluyó inicialmente en los modelos, pero no resultó significativa, por lo que finalmente optamos por no incluirla, dada la correlación con el nivel de estudios, que sí se ha mantenido en los modelos. Por otro lado, ambas variables están igualmente correlacionadas con la profesión de los entrevistados, que también ha sido incluida en los modelos.

${ }^{13}$ No obstante podría plantearse que esta variable tenga un efecto nulo, ya que a los núcleos rurales o pequeños llegaría la misma cantidad de información dado que la televisión es el medio más utilizado para informarse y está bien extendido su uso. En el sentido contrario, aunque a estos núcleos llegan más ayudas directas que llegan de la UE que harían más visible la Unión, incluyendo connotaciones más positivas acerca de lo que significaría apoyar el nuevo proyecto de constitución europea que en núcleos mayores, cabe también la posibilidad de que dichas ayudas sean capitalizadas políticamente como éxitos de la elite local por lo que, de nuevo, el efecto del tamaño del hábitat sería nulo.

EMPIRIA. Revista de Metodología de Ciencias Sociales. N. ${ }^{\circ}$ 14, julio-dicembre, 2007, pp. 13-39. ISSN: 1139-5737 
Tabla 4. Resumen de las variables introducidas en los modelos de regresión logística

\begin{tabular}{lccccc}
\hline \multicolumn{1}{c}{ Nombre } & Tipo* & Valores & $\begin{array}{c}\text { Número } \\
\text { de casos }\end{array}$ & Media & $\begin{array}{c}\text { Desv. } \\
\text { típica }\end{array}$ \\
\hline $\begin{array}{l}\text { Fue a votar el día } \\
\text { de referendo }\end{array}$ & $\mathrm{D}$ & $0-1$ & 1191 & 0,62 & 0,486 \\
$\begin{array}{l}\text { Votó a favor } \\
\text { del Tratado }\end{array}$ & $\mathrm{D}$ & $0-1$ & 666 & 0,76 & 0,43 \\
$\begin{array}{l}\text { Televisión } \\
\text { Prensa }\end{array}$ & $\mathrm{N}$ & $1-5$ & 1151 & - & - \\
Radio & $\mathrm{N}$ & $1-8$ & 1185 & - & - \\
Sexo & $\mathrm{N}$ & $1-5$ & 1177 & - & - \\
Edad & $\mathrm{D}$ & $0-1$ & 1203 & 0,48 & 0,50 \\
Interés política & $\mathrm{I}$ & $18-92$ & 1203 & 44,84 & 16,46 \\
internacional & $\mathrm{O}$ & $1-4$ & 1183 & 2,66 & 0,86 \\
Ideología** & $\mathrm{O}$ & $1-7$ & 648 & 3,75 & 1,29 \\
Partido por el & $\mathrm{N}$ & $1-8$ & 1200 & - & - \\
que votó en 2004 & $\mathrm{O}$ & $1-6$ & 1197 & 3,87 & 1,314 \\
Estudios & $\mathrm{N}$ & $1-12$ & 1183 & - & - \\
Profesión & $\mathrm{N}$ & $1-4$ & 1203 & - & - \\
Comunidad & $\mathrm{O}$ & $1-6$ & 1203 & 2,86 & 1,59 \\
Autónoma & Hábitat & & & &
\end{tabular}

* Tipo: D, dicotómica; N, nominal; O, ordinal; I, intervalo.

** Ordenada de izquierda a derecha.

FUENTE: BRIE 8. ${ }^{\mathrm{a}}$ oleada.

\section{ANÁLISIS: EL IMPACTO DIFERENCIAL DE LOS MEDIOS DE COMUNICACIÓN}

Puesto que las variables dependientes son dicotómicas, se han realizados análisis de regresión logística. En este tipo de análisis los coeficientes de regresión $(\beta)$ no son directamente interpretables en cuanto a su magnitud, aspecto al que no se hará referencia, de forma general, en los apartados siguientes. Sí son interpretables la dirección de la relación entre la variable dependiente y la independiente que captura el coeficiente $\beta$, así como su nivel de significación estadística. Son estos dos aspectos a los que aludirá normalmente en los comentarios que siguen. Cuando, eventualmente, se hagan referencias a las magnitudes, éstas se basaran los odd-ratios (o razón de razones), que pueden ser transformados también en probabilidades ${ }^{14}$.

\footnotetext{
${ }^{14}$ Los odd-ratios se calculan a partir del exponencial de $\beta$.
} 


\section{El impacto de la televisión}

Cuando se controla por el resto de variables que se han incluido en los modelos, la cadena de televisión a través de la cual los ciudadanos se informaron durante el periodo de campaña electoral no tuvo un impacto estadísticamente significativo sobre su probabilidad de acudir a votar (Tabla 5). La preferencia partidista sí resultó significativa, sin embargo. Encontramos que, en comparación con los ciudadanos que habían votado al PP en 2004, quienes votaron al PSOE acudieron a votar el día del referendo en mayor proporción. Resultan significativos también el interés con que los entrevistados siguen la política internacional y la ideología. Así, a mayor interés en la política internacional, mayor probabilidad de haber acudido a votar el día del referendo. Al contrario, cuanto más a la derecha del espectro ideológico se situaban los entrevistados menor fue la proporción en que acudieron a votar. La edad de los entrevistados resulta significativa, aunque no en el sentido esperado. A mayor edad, mayor probabilidad de haber acudido a votar. También votaron con una probabilidad mayor los entrevistados con estudios primarios completos que quienes tenían estudios universitarios o superiores. Por último, en cuanto a la profesión, los empresarios dedicados a actividades comerciales acudieron a votar con una probabilidad menor que las amas de casa, el grupo de referencia. El ajuste del modelo es bueno, con una pseudo $r$ cuadrado de Nagelkerke de 0,26, y una capacidad de clasificar correctamente un $70 \%$ de los casos.

El modelo es capaz de predecir mejor el voto favorable en el referendo sobre la constitución europea, con un pseudo r cuadrado de Nagelkerke de 0,33 , y una capacidad de clasificar correctamente el $78 \%$ de los casos (Tabla 5). Respecto al voto afirmativo en el referendo, la cadena de televisión que los entrevistados miran habitualmente sí resulta significativa. Comparados con quienes no miran ningún canal habitualmente, encontramos que tanto los que miran Antena 3, como los que miran alguna otra cadena de ámbito local o autonómico, votaron a favor con una probabilidad significativamente menor. Las consignas partidistas resultan significativas en el caso del PSOE, cuyos votantes emitieron un voto favorable a la constitución europea con una probabilidad mayor que los votantes del PP, y en el caso de los votantes de partidos de ámbito autonómico que hicieron campaña en contra de la aprobación del tratado constitucional, quienes votaron a favor en menor proporción que los votantes del PP. Aparte de estas variables, sólo la profesión es estadísticamente significativa, de manera que entre los obreros especializados votó a favor un porcentaje mayor de ciudadanos que entre las amas de casa, el grupo de referencia. Otros grupos profesionales no se distinguen significativamente de las amas de casa. Ni el sexo, ni la edad, ni el interés en la política internacional, ni la ideología, ni el hábitat o la comunidad resultan significativos estadísticamente. 
Tabla 5. Probabilidad de que los entrevistados fueran a votar el día del referendo sobre la Constitución Europea, y de que lo hicieran a favor, en función de la cadena de televisión que miran habitualmente

\begin{tabular}{|c|c|c|}
\hline & Ir a votar & Votar a favor \\
\hline \multicolumn{3}{|l|}{ Televisión (categoría de ref. ninguna) } \\
\hline (1) Izquierda & ns & ns \\
\hline (2) Derecha & ns & $-2,048 *$ \\
\hline (3) Autonómica/local & ns & $-2,287 *$ \\
\hline Sexo $(s 01)$ & ns & ns \\
\hline $\operatorname{Edad}(\mathrm{s} O 2)$ & $0,038 * * *$ & ns \\
\hline Interés en la política (s07) & $0,544 * * *$ & ns \\
\hline Ideología (s09) & $-0,167 *$ & ns \\
\hline \multicolumn{3}{|l|}{ Partido al que votó en 2004 (categoría de ref. PP) } \\
\hline (1) PSOE & $0,905 * *$ & $1,041 * *$ \\
\hline (2) IU & ns & ns \\
\hline (3) Ámbito autonómico SI (PNV, CIU, CC) (a) & ns & ns \\
\hline (4) Ámbito autonómico NO (ERC, BNG, CHA, EA) (b) & ns & $-2,026^{* *}$ \\
\hline (5) Otros/blanco & ns & ns \\
\hline (6) Ninguno & $-0,621 *$ & ns \\
\hline (7) No sabe & ns & ns \\
\hline \multicolumn{3}{|l|}{ Estudios (categoría de ref. tercer grado segundo ciclo) } \\
\hline (1) Menos de primer ciclo & ns & ns \\
\hline (2) Primer ciclo completo & $0,818^{*}$ & ns \\
\hline (3) Segundo ciclo primer grado & ns & ns \\
\hline (4) Segundo ciclo segundo grado & ns & ns \\
\hline (5) Tercer ciclo primer grado & ns & ns \\
\hline \multicolumn{3}{|l|}{ Profesión (categoría de ref. amas de casa) } \\
\hline (1) AJ-Directores y mandos superiores & ns & ns \\
\hline (2) AJ-Mandos intermedios & ns & $\mathrm{ns}$ \\
\hline (3) AJ-Capataces, administrativos, representantes & ns & ns \\
\hline (4) AJ-Obreros especializados & ns & $1,661 * *$ \\
\hline (5) AJ-Vendedores, dependientes & ns & ns \\
\hline (6) AJ-Subalternos & ns & ns \\
\hline (7) PR-Empresarios agrarios & ns & ns \\
\hline (8) PR-Empresarios comerciantes & $-0,793 *$ & ns \\
\hline (9) PR-Profesionales liberales & ns & ns \\
\hline (10)PR-Artesanos & ns & ns \\
\hline (11) NT-Estudiantes & ns & ns \\
\hline Comunidad Autónoma (categoría de ref. Andalucía) & & ns \\
\hline (1) Cataluña & ns & ns \\
\hline (2) País Vasco & ns & ns \\
\hline (3) Resto & ns & ns \\
\hline Tamaño del municipio & ns & ns \\
\hline Constante & $-2,740 * *$ & ns \\
\hline $\mathrm{N}$ & 609 & 353 \\
\hline Casos positivos clasificados correctamente & $50 \%$ & $40 \%$ \\
\hline Casos negativos clasificados correctamente & $83 \%$ & $92 \%$ \\
\hline Porcentaje global de casos clasificados correctamente & $70 \%$ & $78 \%$ \\
\hline R cuadrado (Nagelkerke) & 0,26 & 0,33 \\
\hline
\end{tabular}

ns, no significativo

*, estadísticamente significativo con un margen de confianza del 90\%; **, del 95\%; y *** del 99\%.

El tamaño de las muestras para cada modelo, aunque escaso, supera el mínimo de 10 casos por variable independiente introducida en el modelo (mínimo de 340 casos).

(a) Partidos de ámbito autonómico que hicieron campaña a favor del Sí: PNV, CiU, CC

(b) Partidos de ámbito autonómico que hicieron campaña a favor del No: BNG, CHA, EA, ERC

FUENTE: BRIE $8 .^{\mathrm{a}}$ oleada.

EMPIRIA. Revista de Metodología de Ciencias Sociales. ‥ ${ }^{0}$ 14, julio-dicembre, 2007, pp. 13-39. ISSN: 1139-5737 


\section{El impacto de la prensa}

En cuanto a la prensa escrita encontramos que ésta, al contrario que la televisión, sí tuvo un impacto sobre la probabilidad de acudir a votar, aún después de controlar por el resto de variables (Tabla 6). Es decir, quienes leyeron periódicos de izquierda (El País) o periódicos de ámbito local o autonómico, acudieron a votar en mayor medida que quienes no leen la prensa habitualmente. En este sentido, quienes leyeron otros periódicos de derecha (El Mundo, ABC o La Vanguardia) y quienes leyeron otros periódicos (gratuitos o de pago) no se diferenciaron significativamente de quienes no leen la prensa habitualmente (en lo que a su probabilidad de haber acudido a votar el día de referendo se refiere). Además de la lectura de la prensa, resulta significativa también la preferencia partidista de los entrevistados: quienes votaron al PSOE en 2004, acudieron a votar en el referendo con una probabilidad mayor que quienes habían votado al PP. Sin embargo, quienes en 2004 no acudieron a votar tendieron a no hacerlo tampoco en la consulta del referendo, de manera que el porcentaje de entrevistados que se acercó a las urnas fue significativamente menor que entre quienes habían votado al PP (la categoría de referencia).

De manera similar a lo que ocurría en el modelo anterior, son significativas también la edad (a mayor edad, mayor probabilidad de haber acudido a votar) y el interés por la política (a mayor interés, mayor probabilidad de haber acudido a votar). Sin embargo, cuando se controla por la prensa que leen los entrevistados, la ideología no resulta significativa. Por otro lado, con respecto a los entrevistados con mayor nivel de estudios, se observa que quienes tienen estudios primarios o secundarios acudieron a votar con una probabilidad mayor que el grupo de referencia (los ciudadanos con estudios superiores). Finalmente, frente a las amas de casa (el grupo de referencia), los empresarios dedicados a actividades mercantiles también acudieron a votar en menor proporción. Otras profesiones no resultan significativas. El ajuste del modelo es bueno, con un pseudo $r$ cuadrado de Nagelkerke de 0,26 . En conjunto el modelo clasifica correctamente un $72 \%$ de los casos.

$\mathrm{Al}$ igual que ocurría con la televisión, el modelo es capaz de predecir mejor el voto favorable en el referendo sobre la constitución europea, con un pseudo $r$ cuadrado de Nagelkerke de 0,31, y una capacidad de clasificar correctamente el $77 \%$ de los casos (Tabla 6). Sin embargo, al contrario que ocurría con las cadenas de televisión, la prensa que los ciudadanos leen habitualmente no influyó de forma significativa sobre su decisión de votar favorablemente. Es decir, quienes leyeron algún periódico no se distinguen de forma estadísticamente significativa de quienes no leen la prensa habitualmente, una vez controladas el resto de las variables. Las consignas partidistas sí resultan claramente significativas. En el caso del PSOE, quienes lo habían votado en 2004 emitieron un voto favorable a la constitución europea con una probabilidad mayor que quienes habían votado al PP. Quienes habían elegido a partidos de ámbito autonómico que, posteriormente, hicieron campaña en contra de la aprobación del tratado constitucional, 
Tabla 6. Probabilidad de que los entrevistados fueran a votar el día del referendo sobre la Constitución europea, y de que lo hicieran a favor, en función de la prensa que leen habitualmente

\begin{tabular}{|c|c|c|}
\hline & Ir a votar & Votar a favor \\
\hline \multicolumn{3}{|l|}{ Prensa (categoría de referencia ninguno) } \\
\hline (1) Izquierda & $0,768 * *$ & ns \\
\hline (2) Derecha & ns & ns \\
\hline (3) Otros & ns & ns \\
\hline (4) Autonómico/local & $0,518 * *$ & ns \\
\hline $\operatorname{Sexo}(s 01)$ & ns & ns \\
\hline Edad ( $(502)$ & $0,039 * * *$ & ns \\
\hline Interés en la política ( $(507)$ & $0,503 * * *$ & ns \\
\hline Ideología ( $(509)$ & ns & ns \\
\hline \multicolumn{3}{|l|}{ Partido al que votó en 2004 (categoría de ref. PP) } \\
\hline (1) PSOE & $0,819 * *$ & $0,959 * *$ \\
\hline (2) IU & ns & ns \\
\hline (3) Ámbito autonómico SI (PNV, CIU, CC) (a) & ns & ns \\
\hline (4) Ámbito autonómico NO (ERC, BNG, CHA, EA) (b) & ns & $-2,025 * *$ \\
\hline (5) Otros/blanco & ns & $-1,267 * *$ \\
\hline (6) Ninguno & $-0,560 *$ & ns \\
\hline (7) No sabe & ns & ns \\
\hline \multicolumn{3}{|l|}{ Estudios (categoría de ref. tercer grado segundo ciclo) } \\
\hline (1) Menos de primer ciclo & ns & ns \\
\hline (2) Primer ciclo completo & $1,047 * *$ & ns \\
\hline (3) Segundo ciclo primer grado & ns & ns \\
\hline (4) Segundo ciclo segundo grado & $0,593 *$ & ns \\
\hline (5) Tercer ciclo primer grado & $\mathrm{ns}$ & ns \\
\hline \multicolumn{3}{|l|}{ Profesión (categoría de ref. amas de casa) } \\
\hline (1) AJ-Directores y mandos superiores & ns & ns \\
\hline (2) AJ-Mandos intermedios & ns & ns \\
\hline (3) AJ-Capataces, administrativos, representantes & ns & ns \\
\hline (4) AJ-Obreros especializados & ns & $1,508 * *$ \\
\hline (5) AJ-Vendedores, dependientes & ns & $1,638^{*}$ \\
\hline (6) AJ-Subalternos & ns & ns \\
\hline (7) PR-Empresarios agrarios & ns & ns \\
\hline (8) PR-Empresarios comerciantes & $-0,799 *$ & ns \\
\hline (9) PR-Profesionales liberales & ns & ns \\
\hline (10) PR-Artesanos & ns & ns \\
\hline (11) NT-Estudiantes & ns & ns \\
\hline \multicolumn{3}{|l|}{ Comunidad Autónoma (categoría de ref. Andalucía) } \\
\hline (1) Cataluña & ns & ns \\
\hline (2) País Vasco & ns & ns \\
\hline (3) Resto & ns & ns \\
\hline Tamaño del municipio & ns & ns \\
\hline Constante & $-2,945^{* *}$ & ns \\
\hline $\mathrm{N}$ & 621 & 362 \\
\hline Casos positivos clasificados correctamente & $53 \%$ & $36 \%$ \\
\hline Casos negativos clasificados correctamente & $83 \%$ & $92 \%$ \\
\hline Porcentaje global de casos clasificados correctamente & $72 \%$ & $77 \%$ \\
\hline R cuadrado (Nagelkerke) & 0,26 & 0,31 \\
\hline
\end{tabular}

ns, no significativo

*, estadísticamente significativo con un margen de confianza del 90\%; **, del 95\%; y*** del $99 \%$.

El tamaño de las muestras para cada modelo, aunque escaso, supera el mínimo de 10 casos por variable independiente introducida en el modelo (mínimo de 340 casos).

(a) Partidos de ámbito autonómico que hicieron campaña a favor del Sí: PNV, CiU, CC

(b) Partidos de ámbito autonómico que hicieron campaña a favor del No: BNG, CHA, EA, ERC

FUENTE: BRIE $8 .^{\mathrm{a}}$ oleada.

EMPIRIA. Revista de Metodología de Ciencias Sociales. ‥ ${ }^{0}$ 14, julio-dicembre, 2007, pp. 13-39. ISSN: 1139-5737 
votaron a favor con una probabilidad menor que quienes habían votado al PP. Este es también el caso, finalmente, de quienes habían votado a otros partidos o habían votado en blanco en 2004.

Aparte de la preferencia partidista, sólo la profesión es estadísticamente significativa, de manera que entre los obreros especializados, y entre los vendedores y dependientes, votó a favor un porcentaje mayor de ciudadanos que entre las amas de casa, el grupo de referencia. Otros grupos profesionales no se distinguen significativamente de las amas de casa. Ni el sexo, ni la edad, ni el interés en la política internacional, ni la ideología, ni el hábitat o la comunidad resultan estadísticamente significativos.

\section{El impacto de la radio}

También la radio resulta significativa para entender la decisión de acudir a votar de los entrevistados (Tabla 7). Aún después de controlar por el resto de variables, es posible observar que quienes seleccionaron emisoras cuya orientación ideológica es más conservadora, o de derechas, acudieron a votar en menor medida que quienes no oyen ninguna emisora de forma habitual. Este resultado contradice la hipótesis de que todos los que se expusieron a algún medio resultaron movilizados y acudieron a votar en mayor medida que quienes no lo hicieron, y sugiere que algunos medios tuvieron un efecto desmovilizador sobre la ciudadanía. Quienes prefieren emisoras con una orientación más de izquierdas (Radio Nacional para el periodo que estudiamos, o la SER) $\mathrm{u}$ otras emisoras, no se distinguieron significativamente del grupo de control (quienes no oyen habitualmente la radio). Como en el resto de los modelos, la preferencia partidista de los entrevistados tuvo impacto también sobre la decisión de éstos de acudir a votar. Así, quienes en 2004 votaron al PSOE, acudieron a votar el día del referendo en mayor medida que quienes votaron al PP (el grupo de referencia). Por el contrario quienes en 2004 no votaron a ningún partido, continuaron esta tendencia abstencionista el día del referendo, de manera que acudieron a votar en menor medida que quienes en 2004 habían votado al PP.

Como ocurría con los modelos anteriores, son estadísticamente significativos la edad y el interés por la política, de manera que a medida que aumentan ambas variables lo hace también la probabilidad de haber acudido a votar. La ideología, por el contrario, sigue sin ser significativa (una vez controlado el partido y el medio de comunicación). Finalmente, comparados con los ciudadanos con mayor nivel de estudios, quienes cuentan con estudios primarios acudieron a votar en mayor proporción; en tanto que, comparadas con las amas de casa, los empresarios dedicados a actividades mercantiles acudieron a votar con una probabilidad menor. El ajuste del modelo es adecuado con un pseudo $\mathrm{r}$ cuadrado de $\mathrm{Na}$ gelkerke de 0,26 , y capacidad para clasificar correctamente un $72 \%$ de los casos de forma global. 
Tabla 7. Probabilidad de que los entrevistados fueran a votar el día del referendo sobre la Constitución europea, y de que lo hicieran a favor, en función de la emisora de radio que escuchan habitualmente

\begin{tabular}{|c|c|c|}
\hline & Ir a votar & Votar a favor \\
\hline \multicolumn{3}{|l|}{ Radio (categoría de ref. ninguna) } \\
\hline (1) Izquierda & ns & ns \\
\hline (2) Derecha & $-0,623 * *$ & $-1,217 * *$ \\
\hline (3) Otra & ns & ns \\
\hline Sexo $(\mathrm{s} 01)$ & ns & ns \\
\hline Edad (sO2) & $0,041 * * *$ & ns \\
\hline Interés en la política ( $\mathrm{S} 07)$ & $0,560 * * *$ & ns \\
\hline Ideología (s09) & ns & ns \\
\hline \multicolumn{3}{|l|}{ Partido al que votó en 2004 (categoría de ref. PP) } \\
\hline (1) PSOE & $0,837 * *$ & $0,851^{*}$ \\
\hline (2) IU & ns & ns \\
\hline (3) Ámbito autonómico SI (PNV, CIU, CC) (a) & ns & ns \\
\hline (4) Ámbito autonómico NO (ERC, BNG, CHA, EA) (b) & ns & $-2,347 * *$ \\
\hline (5) Otros/blanco & ns & ns \\
\hline (6) Ninguno & $-0,562 *$ & ns \\
\hline (7) No sabe & ns & ns \\
\hline \multicolumn{3}{|l|}{ Estudios (categoría de ref. tercer grado segundo ciclo) } \\
\hline (1) Menos de primer ciclo & ns & ns \\
\hline (2) Primer ciclo completo & $0,883 *$ & ns \\
\hline (3) Segundo ciclo primer grado & ns & ns \\
\hline (4) Segundo ciclo segundo grado & ns & ns \\
\hline (5) Tercer ciclo primer grado & ns & ns \\
\hline \multicolumn{3}{|l|}{ Profesión (categoría de ref. amas de casa) } \\
\hline (1) AJ-Directores y mandos superiores & ns & ns \\
\hline (2) AJ-Mandos intermedios & ns & ns \\
\hline (3) AJ-Capataces, administrativos, representantes & ns & $1,296 * *$ \\
\hline (4) AJ-Obreros especializados & ns & $1,774 * *$ \\
\hline (5) AJ-Vendedores, dependientes & ns & ns \\
\hline (6) AJ-Subalternos & ns & ns \\
\hline (7) PR-Empresarios agrarios & ns & ns \\
\hline (8) PR-Empresarios comerciantes & $-0,808 *$ & ns \\
\hline (9) PR-Profesionales liberales & ns & ns \\
\hline (10) PR-Artesanos & ns & $2,006 * *$ \\
\hline (11) NT-Estudiantes & ns & ns \\
\hline \multicolumn{3}{|l|}{ Comunidad Autónoma (categoría de ref. Andalucía) } \\
\hline (1) Cataluña & ns & ns \\
\hline (2) País Vasco & ns & ns \\
\hline (3) Resto & ns & ns \\
\hline Tamaño del municipio & ns & ns \\
\hline Constante & $-2,731 * *$ & ns \\
\hline $\mathrm{N}$ & 618 & 361 \\
\hline Casos positivos clasificados correctamente & $53 \%$ & $47 \%$ \\
\hline Casos negativos clasificados correctamente & $83 \%$ & $95 \%$ \\
\hline Porcentaje global de casos clasificados correctamente & $72 \%$ & $82 \%$ \\
\hline $\mathrm{R}$ cuadrado (Nagelkerke) & 0,26 & 0,36 \\
\hline
\end{tabular}

ns, no significativo

*, estadísticamente significativo con un margen de confianza del 90\%; **, del 95\%; y*** del $99 \%$.

El tamaño de las muestras para cada modelo, aunque escaso, supera el mínimo de 10 casos por variable independiente introducida en el modelo (mínimo de 340 casos).

(a) Partidos de ámbito autonómico que hicieron campaña a favor del Sí: PNV, CiU, CC

(b) Partidos de ámbito autonómico que hicieron campaña a favor del No: BNG, CHA, EA, ERC

FUENTE: BRIE 8. ${ }^{\mathrm{a}}$ oleada.

EMPIRIA. Revista de Metodología de Ciencias Sociales. ‥ ${ }^{0}$ 14, julio-dicembre, 2007, pp. 13-39.

ISSN: 1139-5737 
La información respecto a qué cadena de radio oyen los ciudadanos habitualmente mejora notablemente la capacidad de predicción del modelo respecto al voto favorable en el referendo sobre la constitución europea. Utilizando esta información se alcanza un pseudo r cuadrado de Nagelkerke de 0,36 , con una capacidad de clasificar correctamente el $82 \%$ de los casos (Tabla 7). La emisora de radio escogida por los entrevistados resulta significativa, de modo que quienes oyen alguna cadena cuya tendencia ideológica puede clasificarse de conservadora, o de derechas, votaron a favor en menor medida que quienes no oyen ninguna emisora de radio habitualmente. Aparte de ésta, únicamente la preferencia partidista y la profesión resultan estadísticamente significativas. Ni el sexo, ni la edad, ni el interés por la política, ni la ideología ni la educación, ni la Comunidad Autónoma de residencia, ni el tamaño del hábitat son estadísticamente significativas cuando se tienen en cuenta la emisora de radio que los entrevistados oyen habitualmente, su preferencia partidista y su profesión.

Respecto a la preferencia partidista, comparados con quienes en 2004 habían votado por el PP, y que constituyen el grupo de referencia, quienes habían votado por el PSOE votaron a favor con una probabilidad mayor, en tanto que quienes habían votado por partidos de ámbito autonómico que, posteriormente, hicieron campaña en contra de la aprobación de la constitución, votaron a favor de ésta en menor medida que los votantes del PP. Por lo que a la profesión de los ciudadanos se refiere encontramos que, comparados con las amas de casa, el grupo constituido por los capataces, administrativos y representantes de empresas votaron a favor con una probabilidad mayor, al igual que los obreros especializados, y los trabajadores manuales y artesanos que trabajan por cuenta propia.

\section{DISCUSIÓN Y CONCLUSIONES: ¿QUÉ IMPACTO TUVIERON REALMENTE LOS MEDIOS DE COMUNICACIÓN?}

¿Qué significa todo lo anterior? ¿Cómo podemos resumir el impacto real de los medios de comunicación sobre el comportamiento electoral de los ciudadanos españoles en el referendo sobre la constitución europea?

Si nos centramos específicamente en el efecto de los medios de comunicación (Tablas 5, 6 y 7), podemos apreciar, respecto a su impacto en la decisión de los ciudadanos de acudir a votar el día del referendo, que los resultados del análisis no confirman las hipótesis planteadas inicialmente en todos los casos. Al menos para la televisión, se observa que quienes miran habitualmente algún canal no acudieron a votar con una probabilidad significativamente mayor que quienes habitualmente no miran la tele. La prensa y la radio que los ciudadanos usan para informarse sí tuvieron un impacto sobre la probabilidad de acudir a votar el día del referendo, pero no en el mismo sentido. Mientras que en caso de la prensa la lectura de determinados periódicos (El País y los de ámbito autonómico o local) incrementó la probabilidad de que los ciudadanos acudieran a votar (comparados con quienes no leen prensa escrita habitualmente), en el caso de 
la radio quienes escucharon determinadas emisoras (COPE y Onda 0 ) acudieron a votar en una proporción aún menor que quienes no escuchan habitualmente la radio. Se dibuja así un panorama complejo en el que no se puede hablar de un único efecto de los medios sobre la ciudadanía, o de una sola dirección de influencia. Parece que tanto la prensa como la radio tendrían un impacto (des)/movilizador más claro que la televisión, quizás por la orientación más comercial y de entretenimiento de esta última.

No es posible ahondar con la profundidad deseable en el significado de estos resultados ya que carecemos de un análisis cualitativo sobre cómo se presentó la campaña electoral en cada uno de estos medios, si se exceptúa prensa -véanse Sampedro, Ruiz Jiménez y Carriço Reis 2005; Ruiz Jiménez y Sampedro 2005. Para el caso de la prensa escrita, se había señalado la posibilidad de que quienes leen habitualmente periódicos cuya cobertura sobre el referendo fue más amplia acudieran a votar con una probabilidad más alta que quienes leen periódicos cuya cobertura fue más limitada. Esta hipótesis se cumple parcialmente, ya que, comparados con quienes no leen prensa, los lectores habituales de El País, el periódico con más informaciones sobre la constitución, acudieron a votar en mayor proporción que los lectores de $E l$ Mundo y $A B C$.

Si comparamos los posibles efectos de refuerzo de diferentes medios con algo más de profundidad de la que permiten las Tablas 5, 6 y 7, encontramos que los refuerzos más importantes se producen entre los votantes del PSOE que leyeron el País (Tabla 8). Es decir, a igualdad de consignas partidistas (las del PSOE), quienes leyeron El País acudieron a votar en mayor medida que el resto. Es interesante también lo que ocurre con la televisión. Aunque encontrábamos que la televisión no tenía un efecto significativo sobre el conjunto de la ciudadanía, sí lo tiene específicamente sobre los votantes del PSOE. Así, entre ellos, quienes miraron algún canal de televisión acudieron a votar con una probabilidad significativamente mayor que quienes no miran la tele habitualmente. Para el caso de los votantes del PSOE se cumpliría, entonces, la hipótesis inicial que se apuntaba al comienzo de esta sección. Pero por otro lado encontramos, además, evidencia de que los mensajes de refuerzo entre partidos y medios de comunicación, ideológicamente afines, funcionan también con respecto a la televisión. Es decir, la probabilidad de que los votantes del PSOE acudieran a votar el día del referendo es mayor en el caso de los televidentes de cadenas con orientación de izquierda (o favorables al gobierno del PSOE): TVE, Tele 5 y Canal +, que en el caso de los televidentes de cadenas con una orientación más conservadora (Antena 3) y de cadenas de ámbito autonómico o local, aunque todos acudieran a votar en mayor proporción que los votantes del PSOE que no miraron la televisión ${ }^{15}$.

${ }^{15}$ No es posible calcular estos efectos de refuerzo para otros partidos debido al reducido número de observaciones, que en varios casos supera al de variables incluidas en los modelos. En otras ocasiones la falta de variabilidad en alguna de las variables hace imposible calcular la matriz de covarianzas.

EMPIRIA. Revista de Metodología de Ciencias Sociales. N. ${ }^{\circ}$ 14, julio-dicembre, 2007, pp. 13-39.

ISSN: $1139-5737$ 
Tabla 8. Impacto de los medios de comunicación sobre la probabilidad de haber acudido a votar en el referendo sobre la Constitución Europea de los votantes del PSOE y PP(a)

\begin{tabular}{lcc}
\hline & Votaron al PSOE en 2004 & Votaron al PP en 2004 \\
\hline Televisión (ref. ninguna) & 1,982 & $\mathrm{~ns}$ \\
(1) Izquierda & 1,671 & $-2,275$ \\
(2) Derecha & 1,686 & $\mathrm{~ns}$ \\
(3) Autonómico/local & 1,619 & - \\
Prensa (ref. ninguna) & $\mathrm{ns}$ & - \\
(1) Izquierda & $\mathrm{ns}$ & - \\
(2) Derecha & $\mathrm{ns}$ & - \\
(3) Otros & & $\mathrm{ns}$ \\
(4) Autonómico/local & $\mathrm{ns}$ & $-0,831$ \\
Radio (ref. ninguna) & $\mathrm{ns}$ & 1,017 \\
(1) Izquierda & $\mathrm{ns}$ & \\
(2) Derecha & (3) Otras &
\end{tabular}

(a) Para calcular los coeficientes de regresión logística que aparecen en cada una de las celdas se han repetido los modelos explicitados en el capítulo para cada grupo de votantes. Para simplificar la presentación de los resultados mostramos únicamente aquellos coeficientes significativos que hacen referencia al impacto de los medios de comunicación, aunque hemos mantenido las variables de control de los modelos en cada uno de los análisis. Debido a problemas con el número de casos introducidos en los modelos, hemos dejado fuera de los análisis las variables de Comunidad Autónoma y tamaño de hábitat, que no resultaban significativas; por otro lado, hemos recodificado el nivel educativo y la profesión de los entrevistados con un número menor de categorías. ns, no significativo

*, estadísticamente significativo con un margen de confianza del 90\%; **, del 95\%; y *** del $99 \%$.

FUENTE: BRIE 8. ${ }^{\text {a }}$ oleada.

Con respecto a los votantes del PP, la interpretación de los resultados es algo más complicada. En análisis anteriores se ha señalado cómo de la prensa escrita conservadora (El Mundo y $A B C$ que eran los periódicos analizados) desaparecieron los mensajes más críticos y negativos con respecto al borrador de constitución europea a medida que se acercaba la fecha de la consulta. En sintonía con esto, encontramos que el periódico que los votantes del PP leen habitualmente no tuvo efectos significativos sobre la probabilidad de que los votantes del PP acudieran a votar el día del referendo (es decir, no encontramos un efecto negativo derivado de la exposición a estos medios). Habíamos apuntado que quizás cabría esperar que quienes leyeran El País acudieran a votar en mayor proporción, pero esta hipótesis no se confirma. Al contrario que la prensa escrita, la radio y la televisión sí tuvieron un impacto claro sobre los votantes del PP. Cabría hipotetizar, quizás para futuras investigaciones que la televisión y la radio llevaron a cabo una campaña algo más negativa que la prensa, y que ésta fue más efectiva tal vez por la facilidad con que la información es consumida por la audiencias a través de esos canales. De este modo, los televidentes de cadenas de televisión con orientación más conservadora (Antena 3) y los oyentes de emisoras de radio con orientación de derechas (COPE y Onda O), acudieron a votar con una pro- 
babilidad menor que quienes no miran la tele u oyen la radio habitualmente. Aunque se carece de los estudios cualitativos que avalen esta hipótesis, cabe apuntar que éstos medios promovieron la abstención (contribuyeron a la desmovilización de la ciudadanía) como forma de castigo al gobierno del PSOE. Por el contrario, los votantes del PP que escucharon otras radios acudieron a votar con una probabilidad mayor que quienes no escuchan ninguna emisora habitualmente.

Por lo que hace al sentido del voto (Tablas 5,6 y 7) cabía espera que quienes utilizaron medios de comunicación más cercanos al PSOE y/o al gobierno votaran a favor en mayor medida que quienes no utilizan habitualmente medio de comunicación alguno para informarse. Esta hipótesis se confirma parcialmente y de forma indirecta; es decir, lo que encontramos es que quienes utilizaron los medios de comunicación más lejanos al PSOE y/o gobierno tendieron a votar a favor en menor proporción. Con respecto a la televisión, encontramos que los ciudadanos que miraron Antena 3 u otro canal del ámbito autonómico o local acudieron a votar en menor medida que quienes no miran la tele habitualmente; por el contrario, quienes miraron algún canal con una orientación más de izquierdas (TVE, Tele 5 o Canal +) no acudieron a votar en una proporción significativamente mayor. También por lo que a la radio se refiere, los oyentes de emisoras con una orientación más conservadora, o de derechas (COPE y Onda 0 ), votaron a favor con una probabilidad menor que quienes habitualmente no oyen la radio. Las emisoras con una orientación ideológica más cercana al gobierno del PSOE no tuvieron, sin embargo, un impacto positivo. Finalmente, cabe señalar, que la prensa escrita no tuvo un impacto significativo sobre el sentido del voto.

En resumen, la conclusión principal, y la más relevante, que cabe extraer de este trabajo, es que los medios de comunicación que los entrevistados utilizan habitualmente para informarse tuvieron un impacto directo sobre su comportamiento electoral en el referendo sobre la constitución europea. Este impacto se mantiene aún después de controlar por el resto de variables que se consideran relevantes para explicar tanto el apoyo a la integración europea, como el comportamiento electoral en general. Especialmente destacable es el hecho de que la influencia de los medios se mantenga después de controlar por la preferencia partidista de los entrevistados, la variable que habitualmente mejor puede predecir el comportamiento electoral. En este caso, los ciudadanos siguieron las consignas partidistas, pero dentro de un mismo grupo de votantes aquellos que eligieron determinado medio para informarse (canal de televisión, periódico o radio) se distinguieron significativamente del resto. La introducción de los medios de comunicación consumidos por los ciudadanos, de forma conjunta con su preferencia partidista y su tendencia ideológica, resuelve además el problema de auto-selección que metodológicamente es el obstáculo principal para este tipo de análisis.

Sin embargo, el hecho de que se haya constatado el impacto de los medios, no significa que ese impacto sea siempre, ni necesariamente, positivo. El patrón 
de influencia no es único. Es decir, comparados con quienes no miran la tele, leen la prensa o escuchan la radio, quienes sí lo hicieron no siempre acudieron a votar en mayor medida que aquellos. $\mathrm{O}$, dicho de otro modo, algunos medios de comunicación tuvieron un efecto desmovilizador sobre la ciudadanía. En el caso del referendo sobre la constitución europea, especialmente algunos medios ideológicamente más cercanos a la derecha (la televisión - Antena 3-y la radio - COPE y Onda 0) parecen haber desincentivado tanto la participación como el voto favorable. El efecto se refuerza entre los votantes del PP específicamente. Como señalábamos anteriormente, estos medios pudieron promover la abstención de forma activa para castigar al partido del gobierno, el PSOE. Se trata de una hipótesis sobre la que habría que profundizar en el futuro. Para ello sería necesario contar con un estudio cuantitativo, que incluyera más variables políticas, y con estudios cualitativos paralelos tanto sobre el contenido y las estrategias de la campaña electoral, como sobre el seguimiento e interpretación de la misma por parte de la ciudadanía.

\section{BIBLIOGRAFÍA}

Álvarez Miranda, B. (1996), El sur de Europa y la adhesión a la Comunidad. Los debates políticos, Siglo XXI, Madrid.

ANDERSON, C. (1998), «When in doubt, use proxies: attitudes toward domestic politics and support for integration», Comparative Political Studies, vol. 31, n. ${ }^{0}$ 5, pp. 569-601.

ARNOLD, R. (2002), «¿Pueden unos ciudadanos poco atentos a la política controlar a sus representantes electos?», Zona Abierta, n. ${ }^{\circ}$ 100/101, pp. 51-72.

BANDUCCI, S., J. KARP y E. LAUf (2004), Framing European institutions: Elite leadership, media coverage and support for European integration, manuscrito, University of Amsterdam, Amsterdam School of Communication Research, ASCoR.

BANDUCCI, S. y H. SEMETKO (2003), Media, mobilization and European elections, manuscrito, University of Amsterdam, Amsterdam School of Communication Research, ASCoR.

Berelson, B., P. LAZASFeld y W. McPhee (1954), Voting: A study of opinion formation in a presidential campaign, University of Chicago Press, Chicago.

BRYNIN, M. y K. NEWTON (2003), «The national press and voting turnout: British general elections of 1992 and 1997», Political Communication, n. ${ }^{\circ}$ 20, pp. 59-77.

Capdevilla, A., L. Gómez y L. Aubia (2005), «Las estrategias partidistas e institucionales ante la Constitución Europea», comunicación presentada en el VII Congreso Nacional de la AECPA, Madrid 2005.

CAREY, S. y J. Burton (2004), «Research note: the influence of the press in shaping public opinion towards the European Union in Britain», Political Studies, n. ${ }^{\circ} 52$, pp. 623 640.

Closa, C. (2001), «Las raíces domésticas de la política europea de España y la presidencia de 2002», Etudes et Recherches (Notre Europe), n. ${ }^{\circ} 16$.

CRESPO MARTínEZ, I. (dir.) (2000-2004), Las campañas electorales y sus efectos en la decisión del voto: las elecciones generales del 2000 en España, Tirant Lo Blanch, Valencia. 
Dalton, R., P. Beck y R. Huckfeld (1998), «Partisan cues and the media: information flows in the 1992 presidential election», American Political Science Review, vol. 91, n. ${ }^{\circ} 1$, pp. 111-126.

Dalton, R. (1996), Citizen politics in Western democracies, Chatham House, Chatham, NJ.

DE VReEse, C. H. (2003), Framing Europe. Television News and European Integration, Amsterdam, Aksant.

Diez-Medrano, J. y P. Gutierrez (2001), «Nested identities: national and European identities in Spain», Ethnic and Racial Studies, vol. 24, n. ${ }^{\circ}$ 5, pp. 753-778.

ENTMAN, R. (1993), «Framing: toward clarification of a fractured paradigm», Journal of Communication, n. ${ }^{\circ} 43$, pp. 51-58.

FERnÁNDEZ Mellizo-Soto, M. (2000), «¿Para que sirven las campañas electorales? Los efectos de la campaña electoral española de 1993», Estudio/Working Paper 2000/148, Instituto de Estudios e Investigaciones de la Fundación Juan March.

GABEL, M. (1998a), Interests and integration: market liberalisation, public opinion and European Union, MI, University of Michigan Press, Ann Arbor, MI.

GABEL, M. (1998b), «Economic integration and mass politics: market liberalisation and public attitudes in the European Union», American Journal of Political Science, vol. 42, n. ${ }^{\circ}$ 3, pp. 936-953.

GELLENI, R, y C. ANDERSON (2000), «The economy, accountability and public support for the president of the European Commission», European Union Politics, vol. 1, n. ${ }^{\circ} 2$, pp. 173-200.

Gómez-Reino, M., I. Llamazares y L. RAmiro (2002), «Euroscepticism and political parties in Spain», comunicación presentada en ECPR Joint Session of Workshop (Turin 2002).

GonzÁlez Álvarez, P. (1998), Clase, voto y estado de bienestar en España (19861996), manuscrito sin publicar, CEACS, Instituto de Estudios e Investigaciones de la Fundación Juan March.

GonZÁleZ, J. (1993), «Clase y apoyo electoral», Sistema, n. ${ }^{\circ} 112$, pp. 41-71.

Hix, S. (2001), «Legislator's ideology or party organizational power? What drives voting behaviour in the European Parliament?», manuscrito presentado en el Seventh Biennial International Conference of the European Community Studies Association, Madison (EEUU).

INGLEHART, R. y J. RABIER (1978), «Economic uncertainty and European solidarity: public opinion trends», Annals of the American Academy of Political and Social Science, n. $^{\circ} 440$, pp. 66-87.

Iyengar, S. y R. Reeves (1997), Do the media govern? Politicians, voters, and reporters in America, Sage, Thousand Oaks, CA.

JAÚREgUI, P. (2001), National Pride and the Meanings of 'Europe: A Comparative Study of Britain and Spain. Ph.D Thesis, European University Institute, Florence.

LanCASter, T. y Michael S. Lewis-BecK (1986), «The Spanish voter: tradition, economics, ideology», The Journal of Politics, n. ${ }^{\circ}$ 48, pp. 648-674.

LaZARsfeld, P., B. Berelson y H. GAUdeT (1944), The people's choice: how the voter makes up his mind in a presidential campaign, Columbia University Press, New York.

LlamaZARES, I. (2005), «The Popular Party and European integration. Re-elaboratin the European program of Spanish conservatism», South European Society and Politic, vol. 10, n. $^{\circ} 2$, pp. $315-332$. 
LUPIA, A. (1994), Shortcuts versus encyclopedias: information and voting behavior in California insurance reform elections, American Political Science Review, vol. 88, n. ${ }^{\circ}$ 1, pp. 63-76.

MoratA. F. (2000), «El Parlamento Europeo: una institución en busca de un papel político», en Am Artínez y M. Méndez (eds.), Las elecciones al Parlamento Europeo, 1999, Tirant Lo Blanch, Valencia, pp. 13-63.

Muñoz Alonso, A. y RosPIR, J. (eds.) (1999), Democracia mediática y campañas electorales, Ariel Comunicación, Barcelona.

Newton, K. y M. BRYNIN (2001), «The national press and party voting in the UE», Political Studies, n. ${ }^{\circ}$ 49, pp. 265-285.

NorRIs, P. (2000), A virtuous circle: political communications in postindustrial societies, Cambridge University Press, Cambridge.

Norris, P., J. Curtice, D. Sanders, M. Scammell y H. Semetko (1999), On message: communicating the campaign, Sage, London.

PAtTerson, T., y R. MCCluRE (1976), The unseeing eye: the myth of television power in national politics, Putnam, New York.

QuinTANILla NAVARRO, M. A. (2000), «Los partidos españoles ante el proceso de integración europea», Revista de Estudios Políticos, n. ${ }^{\circ}$ 108, pp. 307-323.

REIF, K. (1985), «The Second-order National Elections», en K. Reif (ed.), Ten European Elections, Gorver, Aldershot, pp. 1-36.

RuIz JiMÉnEZ, A. M. y NoYA, J. (2004), «Los españoles ante el Tratado Constitucional y el proceso de integración europea», Documentos de Trabajo del Real Instituto Elcano, n. ${ }^{\circ}$ 62/2004.

RuIZ JimÉnEZ, A. M. y V. SAMPEDRo (2005), «La constitución europea en la prensa española de ámbito nacional: un análisis comparativo de El País, El Mundo y $A B C »$, Documentos de Trabajo del Real Instituto Elcano 1/2005

RuIz JimÉneZ, A. y EGEA DE HARO, A. (2007), «Defining the future of the EU: ideology or national environment?», EPNET Kiosk Plus, 5, n. ${ }^{\circ}$ 1, pp. 95-108.

RuIz JiMÉNEZ, A. y EGEA DE HARO, A. (2008 -en prensa), «Spain: Euroscepticism in a pro-European country?», South European Society and Politics, monográfico sobre «Euroscepticism in southern Europe».

SAMPedro, V., A. M. RuIz JimÉnEZ, y B. CARRIÇo ReIZ (2005), «El referendo del Tratado de la UE en la prensa española de referencia: a favor de la Constitución con en contra de la Unión Europea», Documentos de Trabajo del Real Instituto Elcano 20/2005.

SchmitT-BECK, R. (2003), «Mass communication, personal communication and vote choice: the filter hypothesis of media influence in comparative perspective», British Journal of Political Science, n. ${ }^{\circ}$ 33, pp. 233-259.

Seymour-Ure, C. (1997), «Newpapers: editorial opinion in the national press», en P. Norris (ed.), Britain votes, Oxford University Press, Oxford, pp. 78-100.

Swanson, D. y P. MANCINI (eds.) (1996), Politics, media, and modern democracy. An international study of innovations in electoral campaigning and their consequences, Praeger, Westport, $\mathrm{CN}$.

TorReblanCA, J. I. (2001), «La europeaización de la política exterior española», in C. Closa (ed.) La europeización del sistema político español, Madrid, Istmo.

ZALLER, J. (1996), «The myth of massive media impact revived: new support for a discredited idea», en D. Muth, R. Brody y P. Sniderman (eds), Political persuasion and attitude change, University of Michigan Press, Ann Arbor, MI, pp. 121-135.

ZALLER, J.(1992), The nature and origins of mass opinion, Cambridge University Press, Cambridge. 


\section{RESUMEN}

El objetivo principal de este artículo es establecer si el uso que los ciudadanos hicieron de la televisión, la prensa y la radio durante la campaña electoral previa al referendo sobre la constitución europea afectó a su probabilidad de acudir a votar el 20 de febrero de 2005, así como a su probabilidad de votar a favor.

Los resultados obtenidos permiten afirmar que los medios de comunicación jugaron un papel destacado, tanto en lo que a la decisión de ir a votar se refiere, como en la de hacerlo a favor. Las conclusiones a este respecto son sólidas porque se han tenido en cuenta el resto de factores que podrían haber influido sobre ambas decisiones, y además los efectos se comparan para distintos grupos de votantes. No obstante, el impacto de los medios no fue siempre, ni necesariamente, positivo. Algunas cadenas de televisión y emisoras de radio de derecha tuvieron un efecto desmovilizador sobre la ciudadanía.

\section{PALABRAS CLAVE}

Medios de comunicación, comportamiento electoral, UE, constitución europea

\section{ABSTRACT}

The main objective of this article is testing if mass media consumption (television, newspaper and radio) during the electoral campaign previous to the Spanish referendum on the European constitution had any direct effect on citizens turn out on February $20^{\text {th }} 2005$ as well as on their probability of casting a positive vote.

The analyses show that mass media played a relevant role both in the turn out decision, as well as in casting a positive vote. Conclusions are robust because analyses take into account any other relevant variable that might have had an impact upon each decision, and because they compare the effects among different groups of voters. Mass media impact however was not always, not necessarily, positive. Some TV channels and radio broadcastings stations closer to the right had demobilizing effects on citizenry.

\section{KEY WORDS}

Mass media, electoral behaviour, EU, European constitution. 